\title{
Evaluation of an Anthocyanin, Cyanidin 3,5-di-O-glucoside, as an Allelochemical in Red Callus of a Mangrove Sonneratia ovata, Using Protoplast Co-Culture Bioassay Method with Digital Image Analysis
}

\author{
Hamako Sasamoto $^{1}$, Tsukasa Iwashina ${ }^{2}$, Sakae Suzuki $^{3}$, Yoshitaka Azumi ${ }^{1,4}$ \& Yoshiharu Fujii ${ }^{3}$ \\ ${ }^{1}$ Research Institute for Integrated Science, Kanagawa University, Hiratsuka, Kanagawa 259-1293, Japan \\ ${ }^{2}$ Department of Botany, National Museum of Nature and Science, Tsukuba, Ibaraki 305-0005, Japan \\ ${ }^{3}$ Department of International Environmental and Agricultural Science, Tokyo University of Agriculture and \\ Technology, Fuchu, Tokyo 183-8509, Japan \\ ${ }^{4}$ Department of Biological Sciences, Faculty of Sciences, Kanagawa University, Hiratsuka, Kanagawa 259-1293, \\ Japan \\ Correspondence: Hamako Sasamoto, Research Institute for Integrated Science, Kanagawa University, Hiratsuka, \\ Kanagawa 259-1293, Japan. Tel: 81-463-59-4111. E-mail: sasamoto@ynu.ac.jp
}

Received: February 20, $2018 \quad$ Accepted: March 10, $2018 \quad$ Online Published: March 29, 2018

doi:10.5539/jps.v7n2p1

URL: https://doi.org/10.5539/jps.v7n2p1

\begin{abstract}
Protoplasts isolated from red callus of a mangrove, Sonneratia ovata were co-cultured with recipient lettuce protoplasts to examine the allelopathic activities. Protoplasts were isolated with Cellulase R10 and Driselase 20 in $0.6 \mathrm{M}$ mannitol solution and purified by density gradient centrifugation on $0.6 \mathrm{M}$ sucrose. Protoplasts were co-cultured in $50 \mu \mathrm{L}$ of liquid Murashige and Skoog's (MS) basal medium containing $1 \mu \mathrm{M}$ 2,4-dichlorophenoxyacetic acid and $0.1 \mu \mathrm{M}$ benzyladenine and $0.6 \mathrm{M}$ mannitol solution in a 96-well culture plate. Protoplast density ranged from $5 \times 10^{3} / \mathrm{mL}$ to $10^{5} / \mathrm{mL}$. Cell division of lettuce protoplasts was strongly inhibited by addition of $S$. ovata protoplasts, and non-spherical cell enlargement was slightly inhibited. By contrast, digital image analysis of scanned 96-well culture plates revealed no inhibition in accumulation of yellow color in lettuce protoplasts. An anthocyanin, cyanidin 3,5-di- $O$-glucoside (cyanin), was identified and its content in the red callus was ca. $1 \mathrm{mM}$ of fresh weight. The effects of cyanin on the growth of lettuce protoplasts at three stages were similar to those of red $S$. ovata protoplasts. From these results, cyanin was most likely the allelochemical contained in red callus of $S$. ovata. The allelopathic activity of cyanin was compared with that of other putative allelochemicals in several plant materials, using the protoplast co-culture method with digital image analysis.
\end{abstract}

Keywords: allelopathy, anthocyanin, mangrove plant, protoplast culture, image analysis

\section{Introduction}

\subsection{Salt Tolerance and Allelopathy of Mangrove Cultured Cells}

Mangrove plants are mainly tree species growing in brackish water of different salinity in tropical and subtropical areas (Tomlinson, 1986; Spalding et al., 2010). Strong salt tolerance at the cellular level of several mangrove plants, growing in the coastal region with a high salinity, has been found using tissue cultured cells and protoplasts (Kawana \& Sasamoto, 2008; Hayashi et al., 2009; Yamamoto et al., 2011; Hasegawa et al., 2013).

A survival strategy of the upstream-grown mangrove plants could be the inhibition of the growth of neighboring plants through production of allelochemicals (Hasegawa, 2014; Hasegawa et al., 2014). Allelopathic activities of many non-mangrove test plants using lettuce seedlings as recipients have been studied by the in vitro bioassay sandwich method (Fujii, 2000; Takemura et al., 2013). However, studies on allelopathy of mangrove plant cells were started only recently, after the in vitro bioassay method of allelopathy using tissue cultured cells and protoplasts was developed. 


\subsection{Protoplast Co-culture Method for Bioassay of Allelopathy}

The protoplast co-culture method for in vitro bioassay of allelopathy using lettuce protoplasts as the recipient was recently developed for examining not only herbaceous plant species, e.g., leguminous Mucuna pruriens (Sasamoto et al., 2013) and Vicia villosa (Sasamoto et al., 2015b), and Arabidopsis thaliana (Sasamoto et al., 2017b), but also woody and tree plant species (Hasegawa et al., 2014; Inoue et al., 2015; Mori et al., 2015; Ogita \& Sasamoto, 2017; Fujise et al., 2018) including a tropical invader leguminous tree plant, Leucaena leucocephala (Mori et al., 2015). Using the same lettuce protoplast co-culture method, the inhibitory effects of putative allelochemicals in plants, e.g., L-DOPA (Sasamoto et al., 2013); purine alkaloids, caffeine metabolites (Sasamoto et al., 2015a); pyridine alkaloids, mimosine (Mori et al., 2015); nicotinic acid, nicotinamide and trigonelline (Sasamoto \& Ashihara, 2014); isoflavonoid, rotenone (Inoue et al., 2015); abscisic acid and coumarin (Fujise et al., 2018), were determined.

\subsection{Allelopathic Activities of Sonneratia Mangrove Cells}

After development of the protoplast co-culture method, an inverse relationship was found between allelopathic activity and salt tolerance using dark-grown suspension cultured cells of three Sonneratia mangrove species, $S$. alba, S. ovata, and S. caseolaris (Hasegawa et al., 2014). Salt tolerance to $\mathrm{NaCl}$ and allelopathic activity of suspension cells of $S$. ovata were in-between those of $S$. alba and $S$. caseolaris. However, mangrove cultured cells have not been examined for putative allelochemicals, except for a leguminous tree mangrove, Derris indica, which contained rotenone an isoflavonoid, in roots, but its content in cultured cells remains unknown (Inoue et al., 2015).

\subsection{Allelopathic Activities of Red Callus Culture of S. ovata and Anthocyanins}

Red calluses were induced from hypocotyls, cotyledons and roots of $S$. ovata, and could be sub-cultured in a light condition (Hasegawa, 2014). In contrast, from S. alba and S. caseolaris, no red callus strain was obtained (Sasamoto et al., 2017c). Anthocyanins are natural plant pigments (Takeda et al., 2013), but allelopathic activities of anthocyanins have not been reported.

Here, we identified an anthocyanin in red $S$. ovata callus cultured in the light condition, as cyanidin 3,5-di-O-glucoside (cyanin). Then, using the protoplast co-culture bioassay method, allelopathic activities of protoplasts of red callus of $S$. ovata and the putative allelochemical, cyanin, were investigated. In addition, we used digital image analysis developed recently for protoplast co-culture method of allelopathy, to quantify the effects on the yellow color accumulation during lettuce protoplasts growth (Ogita \& Sasamoto, 2017; Sasamoto et al., 2017a-c). Other allelopathic plants and their putative allelochemicals were also investigated by the protoplast-co-culture bioassay method for comparison.

\section{Method}

\subsection{Materials}

Red callus culture of $S$. ovata was induced from cotyledons of Sonneratia ovata in the MS (Murashige \& Skoog, 1962) basal medium containing $0.1 \mu \mathrm{M}$ of 2,4-dichlorophenoxyacetic acid (2,4-D), 3\% sucrose (Hasegawa, 2014), and sub-cultured on agar medium ( $0.8 \%$ agar) of the same composition for induction, in a light condition (16 hrs photoperiod, $90-140 \mu \mathrm{mol} \mathrm{m} \mathrm{s}^{-1}$ ) at $25^{\circ} \mathrm{C}$.

Lettuce seedlings were cultured as described previously (Sasamoto et al., 2013). Briefly, lettuce seeds (Great Lakes) were sterilized with $1.5 \% \mathrm{NaClO}$ solution for $15 \mathrm{~min}$ and washed three times with autoclaved water. They were aseptically cultured on $0.8 \%$ agar medium in the light condition for $6-8$ days at $25^{\circ} \mathrm{C}$.

\subsection{Identification and Quantification of Anthocyanin in Red Callus}

\subsubsection{Isolation and Identification of Anthocyanin in Red Callus}

Red callus (fresh weight, $15.8 \mathrm{~g}$ ) was extracted with methanol/formic acid (92:8). After concentration, the extract was applied to preparative paper chromatography (PC) using a solvent system, BAW ( $n$-butanol/acetic acid/water $=4: 1: 5$, upper phase). The isolated anthocyanin was purified by Sephadex-LH-20 column chromatography using solvent system, methanol/water/formic acid (70:25:5). Analytical high performance liquid chromatography (HPLC) of the isolated anthocyanin was performed with a Shimadzu HPLC systems using L-column2 ODS column (I.D. $6.0 \times 150 \mathrm{~mm}$, Chemicals Evaluation and Research Institute) at a flow-rate of 1.0 $\mathrm{mL} / \mathrm{min}$, detection wave-length of $530 \mathrm{~nm}$, and eluent was acetonitrile/phosphoric acid/acetic acid/water (8:3:8:81). Liquid chromatograph-mass spectra (LC-MS) was measured with Shimadzu LCMS-2010 EV systems using L-column2 ODS column (I.D. $2.1 \times 100 \mathrm{~mm}$ ) at a flow-rate of $0.2 \mathrm{~mL} / \mathrm{min}$, detection wavelength of 530 $\mathrm{nm}$, ionizing voltage, $4.5 \mathrm{kV}$ for $\mathrm{ESI}^{+}$, and eluent was acetonitrile/water/formic acid (5:90:5). Acid hydrolysis 
was performed in $12 \% \mathrm{HCl}$ at $100^{\circ} \mathrm{C}$ for $30 \mathrm{~min}$. After cooling, iso-amyl alcohol was added in mother liquor, and anthocyanidin and sugar were obtained from the organic layer and aqueous layer, respectively. Anthocyanidin was characterized by HPLC using the authentic sample for comparison. Sugar was identified by PC with the authentic sample for comparison using solvent systems, BBPW ( $n$-butanol/benzene/pyridine/water $=5: 1: 3: 3$ ) and BTPW ( $n$-butanol/toluene/pyridine/water $=5: 1: 3: 3$ ). Sugar spot was visualized by spraying to the chromatogram with $1 \%$ methanolic aniline hydrochloride and then heating.

\subsubsection{Quantification of Anthocyanin in Red Callus}

Red callus (100 mg fresh weight) was extracted in $1 \mathrm{~mL}$ of $2 \%$ trifluoroacetic acid (TFA) / methanol for more than $4 \mathrm{hrs}$ in a dark condition at $4^{\circ} \mathrm{C}$. The extracts were centrifuged at $13,000 \mathrm{rpm}$ for $10 \mathrm{~min}$ at $4{ }^{\circ} \mathrm{C}$, and the supernatant were filtered through a $13 \mathrm{~mm}$ syringe filter with a PVDV membrane (polyvinylidene difluoride, $0.45 \mu \mathrm{m}$, Whatman, Japan) and used for HPLC analysis. Anthocyanin was identified using a Shimadzu HPLC system (Shimadzu Co., Kyoto, Japan) equipped with a Prominance HPLC system (LC-20AD pumping system, a SIL-20AHT autosampler, SPD-M20A diode array detector $(200 \sim 600 \mathrm{~nm}$ detection range). A Shim-pack VP-ODS column ( $4.6 \mathrm{~mm} \Phi \times 150 \mathrm{~nm}$, Shimadzu, Co., Kyoto, Japan) at $40^{\circ} \mathrm{C}$ was used for chromatography to separate anthocyanin species using $0.5 \%$ TFA in ultrapure water as solvent A and $0.1 \%$ TFA in acetonitrile as solvent B. The solvent gradient was \%B, initial 8\%; $50 \mathrm{~min}, 15 \%$; $60 \mathrm{~min}, 30 \%$; $65 \mathrm{~min}, 60 \%$ and $75 \mathrm{~min}, 65 \%$ (run time $75 \mathrm{~min}$ ); at flow rate of $1 \mathrm{~mL} / \mathrm{min}$ and injection volume of $10 \mu \mathrm{L}$. Cyanidin 3-O-glucoside (Cy-3-glu, Wako Chemical Co. Ltd.) was used to form a standard curve and the value of each anthocyanin present was calculated in $\mu \mathrm{g}$ Cy-3-glu equivalents/g fresh weight. Peaks were identified by comparison with a standard of Cy-3-glu and retention times of blueberry-fruit extracts (Ogawa et al., 2008).

\subsection{Protoplasts Isolation and Purification}

Red purple callus of S. ovata at 1.5-2 months of culture were incubated in 1\% each of Cellulase R10 (Yakult) and Driselase 20 (Kyowa Hakko Kogyo Co. Ltd.) in $0.6 \mathrm{M}$ mannitol solution, shaking at $80 \mathrm{rpm}$ speed for $24 \mathrm{hrs}$ This combination of enzymes was selected from 24 combinations in a preliminary experiment using six kinds of cell wall degrading enzymes as described previously (Sasamoto et al., 1997; Azumi \& Sasamoto, 2017). After filtration through $42 \mu \mathrm{m}$ mesh, protoplasts were purified by floatation in density gradient on $0.6 \mathrm{M}$ sucrose solution and washed with mannitol solution by centrifugation at $100 \mathrm{~g} 5 \mathrm{~min}$ three times.

Lettuce cotyledon protoplasts were isolated as described previously (Sasamoto et al., 2013). Briefly, protoplasts of lettuce were isolated in 1\% each of Cellulase RS (Yakult) and Macerozyme R10 (Yakult) in 0.6 M mannitol. Protoplasts were passed through a $63 \mu \mathrm{m}$ nylon mesh and purified with mannitol solution by centrifugation at $100 \mathrm{~g} 5 \mathrm{~min}$ three times.

\subsection{Protoplasts Co-Culture of S. ovata and Lettuce}

Protoplasts were counted using a hemocytometer. Five $\mu \mathrm{L}$ each of protoplasts in $0.6 \mathrm{M}$ mannitol solution was put in $50 \mu \mathrm{L}$ of liquid MS medium containing $1 \mu \mathrm{M}$ of 2,4-D and $0.1 \mu \mathrm{M}$ of benzyladenine (BA), and $3 \%$ sucrose and $0.6 \mathrm{M}$ mannitol in a well of 96-well culture plate (Falcon 3075). Final protoplast densities of $S$. ovata were $1.5 \times 10^{3} / \mathrm{mL}$ to $10^{5} / \mathrm{mL}$, and of lettuce were $6 \times 10^{3} / \mathrm{mL}$ to $10^{5} / \mathrm{mL}$. The protoplasts were cultured at $28^{\circ} \mathrm{C}$ in a humid incubator $\left(\mathrm{CO}_{2}\right.$-incubator without the supply of $\mathrm{CO}_{2}$, APC-30DR, ASTEC Co. Ltd.). The non-spherically enlarged lettuce protoplasts and divided cells were counted under an inverted microscope (Olympus IX-71) after 5 days of culture. The numbers of divided protoplasts including colonies composed of more than 4 cells were counted after 12 days of culture. Percentages of control without addition of $S$. ovata protoplasts were calculated and averaged with standard error (SE) at different densities of lettuce protoplasts.

\subsection{Effects of Cyanin on Lettuce Protoplasts}

Cyanin (cyanidin 3,5-di-O-glucoside chloride, PhytoLab, Kanto Chemical Co. Ltd.) was dissolved in water (1 $\mathrm{mM}$ ) and filter sterilized (Millex-GV, $0.22 \mu \mathrm{m}$, Millipore): $25 \mu \mathrm{L}$ was added to $25 \mu \mathrm{L}$ of double concentrations of each medium composition described in the co-culture method except for mannitol. The final mannitol concentration was $0.4 \mathrm{M}$ instead of $0.6 \mathrm{M}$. The numbers of non-spherically enlarged lettuce protoplasts and divided cells were counted after 4 days of culture. The numbers of divided cells and colonies were counted after 8 days of culture. Percentages of control without cyanin were calculated and averaged with SE at different lettuce protoplast densities.

\subsection{Digital Image Analysis}

Image analysis of yellow color accumulation of lettuce protoplasts after protoplast co-culture was performed as described previously (Ogita \& Sasamoto, 2017; Sasamoto et al., 2017a-c). Digital image of a 96-well culture plate was scanned using a scanner (Epson GTX-970) after 27-35 days of culture. Image analysis by software 
Image J (NIH, Rasband, 1997-2016) was performed. An image of blue channel (jpg file) was selected. A horizontal straight line was drawn at the center of the wells. The plot profile of the line was analyzed. The data of the blue plot values were saved as an excel file. In excel software, the average of blue plot values were determined for each well. The yellow value was converted by deduction of each averaged blue value from the highest blue value (control). The yellow values were deduced at each density of $S$. ovata protoplasts or each concentration of cyanin themselves. The \% yellow value of control without $S$. ovata protoplasts or cyanin was calculated at each lettuce protoplast density. Finally, the percentages of control were averaged with SE at different cell densities of lettuce $\left(6 \times 10^{3} / \mathrm{mL}\right.$ to $\left.10^{5} / \mathrm{mL}\right)$.

\section{Results and Discussion}

\subsection{Red Callus of S. ovata}

Red calluses were induced from all tissues of germinated seedlings, i.e., hypocotyls, cotyledons and roots, of $S$. ovata (Hasegawa, 2014), and red calluses originating from cotyledons could be sub-cultured for more than four years in the light condition. Figure 1 shows red to red-purple $S$. ovata callus originating from cotyledons. After sub-culture at two-week intervals, the red-purple color was maintained during growth. However, when the sub-culture interval was prolonged, the red color diminished after transfer to fresh medium, and red-purple color increased after one month of culture, before browning proceeded.

Several tissue parts of Sonneratia plants were red, e.g., small parts of flowers (Minagawa et al., 2016), and transiently pink seeds of $S$. alba when imbibed in water, but not the seeds of $S$. ovata (Sasamoto et al., 2017c). However, no red callus strain was obtained from young seedlings of $S$. alba and $S$. caseolaris even in the light condition. On the other hand, green callus cultures were obtained from $S$. alba in the light condition (Kawana et al., 2007), and blue-green suspension cultured cells were obtained from S. caseolaris in the dark (Hasegawa et al., 2014; Sasamoto et al., 2017c). Red callus might be unique to S. ovata.

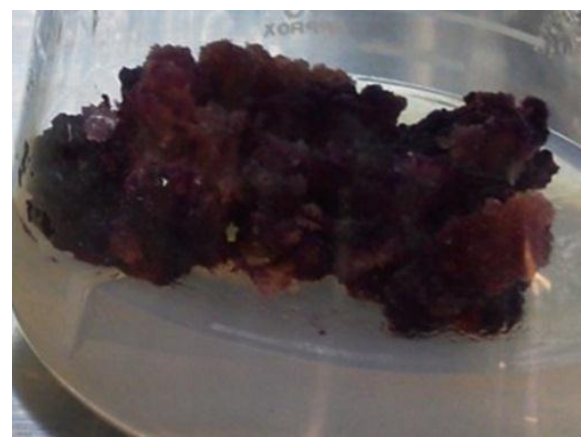

Figure 1. Red-purple Sonneratia ovata callus at 1.5 months of culture in a $100 \mathrm{ml}$ flask

\subsection{Identification and Quantification of Anthocyanin in Red Callus of S. ovata}

The anthocyanin was obtained as a pure red solution (see the subsection 2.2.1). Cyanidin and glucose were liberated by acid hydrolysis of this anthocyanin. Since the molecular ion peak, $m / z 611[\mathrm{M}]^{+}$appeared by LC-MS, 2 mol glucose was shown to be attached to cyanidin. Finally, anthocyanin was identified as cyanidin 3,5-di-O-glucoside (cyanin) (Figure 2), which was not found in the fruit of S. ovata (Wu et al., 2009), by HPLC using the authentic sample for comparison from the flowers of Dahlia variabilis (Hayashi, 1933).

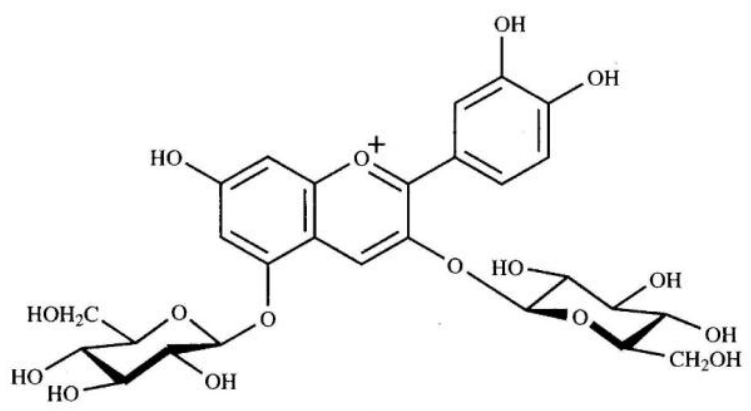

Figure 2. Cyanidin 3,5-di-O-glucoside (cyanin) 
Figure 3 shows the HPLC profile of anthocyanin in red callus of S. ovate (see the subsection 2.2.2). The peak at 7 min was the main peak of cyanin, which was very rapidly eluted than those of the peaks of blueberry extracts (Ogawa et al., 2008), which contained delphinidin 3-O-galactoside (9 min). The calculated content of the main peak was $573 \mu \mathrm{g}$ eq./g fresh weight. The cyanin content in the red callus of $S$. ovata was ca. $1 \mathrm{mmole} / \mathrm{kg}$ fresh weight.

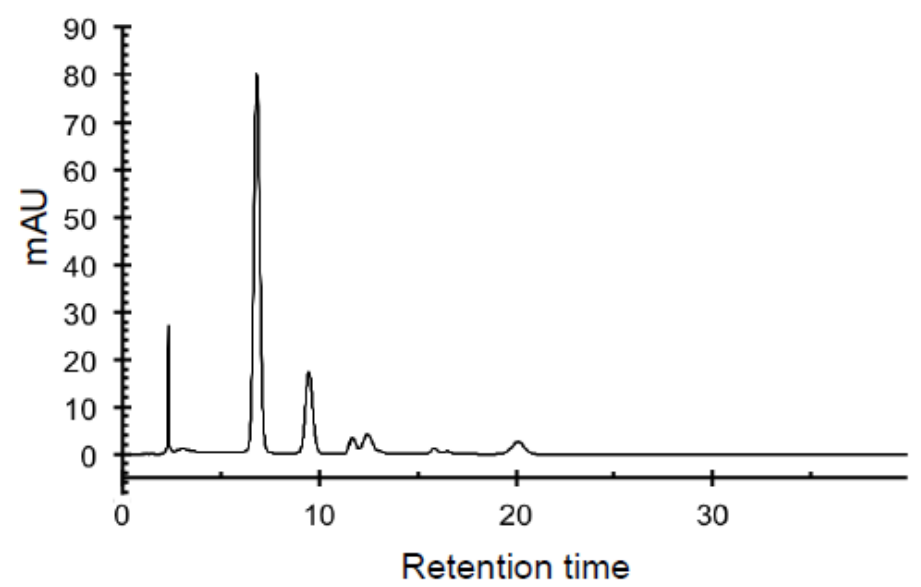

Figure 3. HPLC profile of an extract of $S$. ovata red callus

\subsection{Isolation and Purification of Protoplasts of S. ovata Callus}

The cell-wall-degrading enzyme combination of Cellulase R10 and Driselase 20 was effective for protoplast isolation from red-purple-colored callus of $S$. ovata. The strong enzyme combination consisting of Cellulase RS, Hemicellulase and Driselase 20, which was used for dark-grown suspension cells of S. ovata (Hasegawa et al., 2014), was not used for the red callus in the 24-well method (Azumi \& Sasamoto, 2017; Sasamoto et al., 1997). The density gradient centrifugation method for purification of intact protoplasts was effective for separation from broken protoplasts. Such a method was also effective for protoplast purification of callus culture of mangrove plants, Avicennia alba (Tsuchiya et al., 2013), and suspension cells of S. alba (Hasegawa et al., 2014). As dark-grown $S$. ovata suspension cells could be purified by centrifugation (Hasegawa et al., 2014), cell wall components of red callus might be different from dark-grown suspension cells. The protoplast precipitate of $S$. ovata red callus was pink.

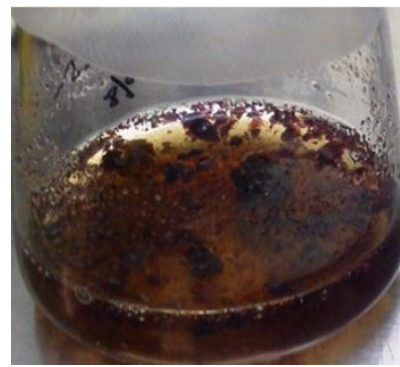

(A)

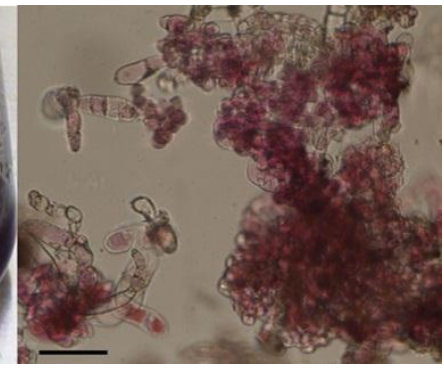

(B)

Figure 4. Red cell clumps of $S$. ovata callus, after $10 \mathrm{~min}$ in enzyme solution in a $100 \mathrm{ml}$ flask (A) and the cell clumps under an inverted microscope (B). Bar $=100 \mu \mathrm{m}$

\subsection{Protoplasts Co-Culture of S.ovata Red Callus and Lettuce}

Figure 5 shows green lettuce protoplasts $30-50 \mu \mathrm{m}$ in diameter and red to light red $S$. ovata protoplasts $15-25 \mu \mathrm{m}$ in diameter. The size of $S$. ovata was similar to those of $S$. caseolaris suspension cells, but smaller than protoplasts of dark-grown $S$. ovata suspension cells (30-50 $\mu \mathrm{m}$ diameter, Hasegawa et al., 2014). The vacuole of protoplasts was red. 


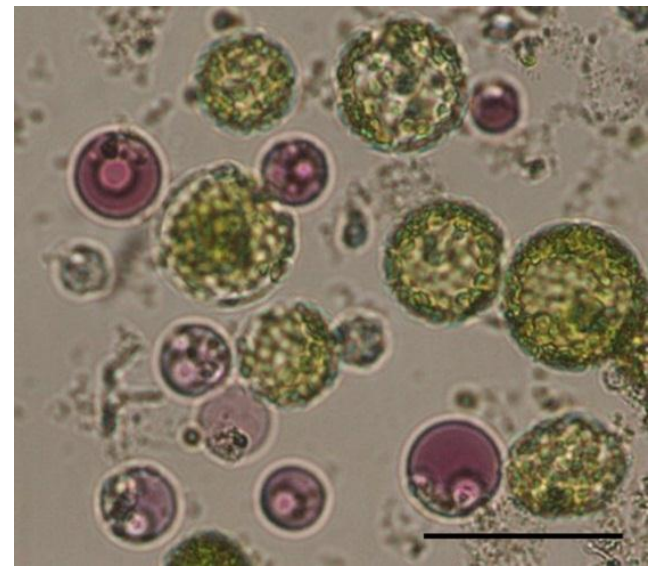

Figure 5. Protoplasts of S. ovata at $5 \times 10^{4} / \mathrm{mL}$ and of lettuce at $10^{5} / \mathrm{mL}$ in MS basal medium containing $1 \mu \mathrm{M}$ of 2,4-D and $0.1 \mu \mathrm{M}$ of BA, $3 \%$ sucrose and $0.6 \mathrm{M}$ mannitol on the first day of culture. Bar=50 $\mu \mathrm{m}$

Though protoplasts of $S$. ovata changed (non-spherical shape change, cell wall formation) after several days of culture, they did not enlarge or divide well in the medium of co-culture and their red color decreased. The change in lettuce protoplasts was well distinguished from their size and divisions under an inverted microscope (Sasamoto \& Ashihara, 2014; Sasamoto et al., 2015a).

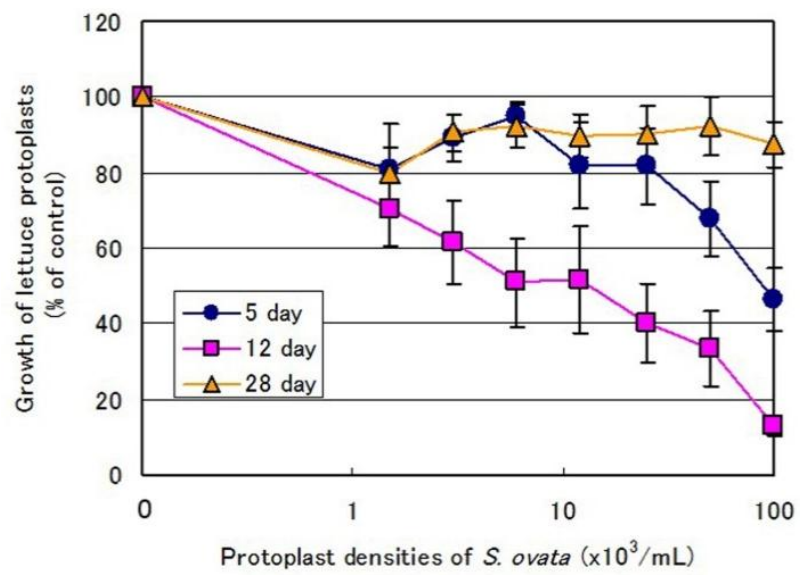

Figure 6. Effects of $S$. ovata protoplasts on growth of lettuce protoplasts by the protoplast co-culture method. Basal medium is shown in Figure 5. Numbers of non-spherically enlarged and divided lettuce protoplasts were counted after 5 or 12 days of co-culture. Digital image analysis of yellow accumulation was performed after 28 days of co-culture. Data were described as \% of control without S. ovata protoplasts and averaged with SE at lettuce protoplast densities of $6-100 \times 10^{3} / \mathrm{mL}$

Figure 6 shows the effects of $S$. ovata protoplasts on the growth of lettuce protoplasts. At 5 days of co-culture, non-spherical enlargement of lettuce protoplasts was clearly inhibited by the addition of $5 \times 10^{4} / \mathrm{mL}$ or more of $S$. ovata protoplasts. After 12 days of co-culture, cell division of lettuce protoplasts was inhibited by the addition of S. ovata protoplasts at low $\left(3 \times 10^{3} / \mathrm{mL}\right)$ to high protoplast densities (up to $10^{5} / \mathrm{mL}$ ). Formation of colonies composed of more than 4 cells was more strongly inhibited than early cell division (data not shown). Such inhibition pattern was different from that of Derris indica which showed the same inhibitory patterns at two stages of growth (cell enlargement stage and division stage).

Allelopathic activity of protoplasts at low densities of red callus of $S$. ovata sub-cultured in the light condition was stronger than that of dark-grown suspension cells of S. ovata (Hasegawa et al., 2014), which might be related to the differences of allelochemical(s) between the two cell lines of S. ovata.

By contrast, no inhibition was observed on the yellow color accumulation in lettuce protoplasts after 28 days of co-culture (Figure 6). Data were similar to those at 35 days of co-culture (data not shown). The yellow substance 
formed during the growth of lettuce protoplasts was identified as a carotenoid (Sasamoto et al., 2017a). Effects on the yellow color accumulation in protoplasts by the co-culture method were very recently investigated using protoplasts of several plant species, e.g., Vicia villosa (Sasamoto et al., 2015b); bamboo species (Ogita \& Sasamoto, 2017); Arabidopsis thaliana (Sasamoto et al., 2017b). In these plants, the yellow color accumulation was inhibited, though the inhibition was weaker than that at other growth stages (cell wall formation and cell divisions). These results are different from the results we obtained using red $S$. ovata protoplasts, which did not inhibit the yellow color accumulation at a protoplast density of up to $10^{5} / \mathrm{mL}$. Specific allelochemical(s) in red callus of $S$. ovata might be considered for this phenomenon.

\subsection{Allelopathic Activities of Cyanin}

Figure 7 shows the effects of an anthocyanin, cyanin on the growth of lettuce protoplasts. The inhibitory effect at the cell division stage was stronger than that at the non-spherically cell enlargement stage (cell wall formation). By contrast, yellow color accumulation was not inhibited after 27 days of co-culture. The same pattern of inhibition as shown in Figure 6 was obtained at a high concentration of cyanin.

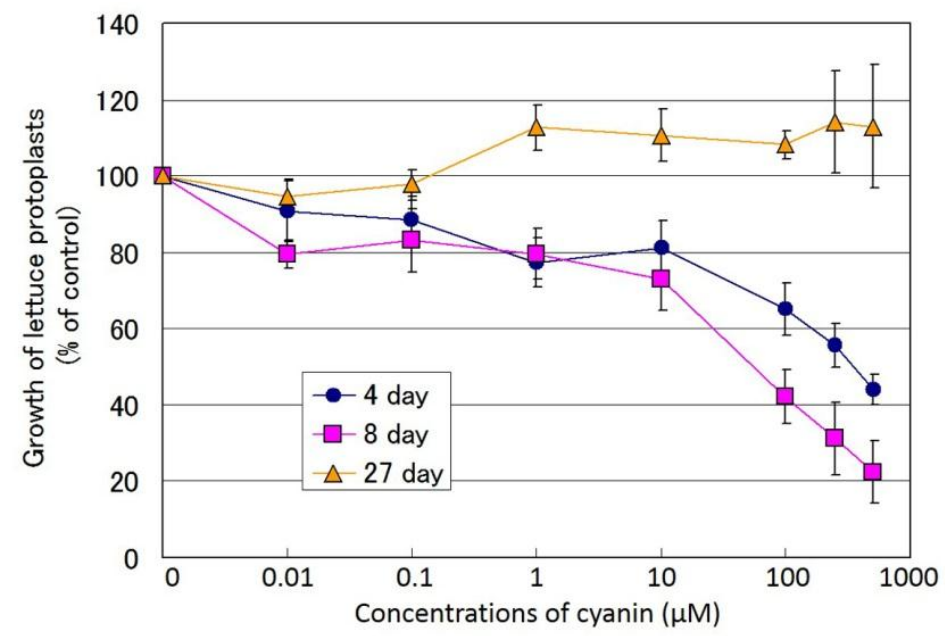

Figure 7. Effects of cyanin on growth of lettuce protoplasts by the protoplast co-culture method. Basal medium was the same as in Figure 5 except for $0.4 \mathrm{M}$ mannitol. Numbers of non-spherically enlarged and divided lettuce

protoplasts were counted after 4 or 8 days of co-culture. Digital image analysis of yellow accumulation was performed after 27 days of co-culture. Data were described as \% of control without cyanin and averaged with SE at lettuce protoplast densities of $6-90 \times 10^{3} / \mathrm{mL}$

As the cyanidin 3,5-di- $O$-glucoside chloride could not be dissolved directly in the co-culture medium, the $1 \mathrm{mM}$ solution was dissolved in water and filter-sterilized before mixing with medium of double concentrated composition. By the addition of cyanidin 3,5-di-O-glucoside chloride, the initial medium $\mathrm{pH}$ 5.2-5.4 was slightly reduced $(4.9,4)$ at high concentrations $(250$ and $500 \mu \mathrm{M})$. However, the difference was not as large as the reduction in $\mathrm{pH}$ (4.5-4.0) after culture of protoplasts of lettuce. This indicated that the inhibition of cell division and absence of inhibition of yellow accumulation at $100 \mu \mathrm{M}$ was not the effect of a low $\mathrm{pH}$.

Though lettuce protoplasts can grow in a wide range of osmotic conditions, i.e., 0.4 to $0.8 \mathrm{M}$ mannitol, lettuce protoplasts grow better in $0.4 \mathrm{M}$ mannitol solution than in $0.6 \mathrm{M}$ mannitol solution. Four days and 8 days were selected for counting the non-spherical enlargement and cell divisions, respectively in $0.4 \mathrm{M}$ mannitol solution, as in the protoplast co-culture with Derris indica and rotenone (Inoue et al., 2015).

3.6 Anthocyanin is the Allelochemical of S. ovata Red Callus Evaluated Using Protoplast Co-Culture Method with Digital Image Analysis

This is the first report on allelopathic activity of red colored callus of a mangrove $S$. ovata containing an anthocyanin, cyanidin 3,5-di- $O$-glucoside (cyanin). Patterns of inhibition at three different stages of growth of lettuce protoplasts, i.e., cell wall formation, cell division and yellow color accumulation, were similar in both $S$. ovata protoplasts and cyanin. Content of cyanin in red callus of S. ovata (ca. $1 \mathrm{mM}$ ) was sufficient for inhibition of lettuce protoplast growth (Figure 7). Though the content of cyanin in S. ovata protoplasts was not investigated, a higher concentration in protoplasts is expected without cell walls. Cyanin is most likely the allelochemical in 
red callus of $S$. ovata, and it is the only allelochemical evaluated in Sonneratia mangrove species using the protoplast co-culture method.

Inhibitory effect of cyanin on cell divisions was weak compared with another putative allelochemical of a mangrove Derris indica, rotenone, which is known in the plant roots. However, no direct investigation was made on its content in the suspension cells of $D$. indica (Inoue et al., 2015). The inhibition by cyanin was stronger than that by caffeine (Sasamoto et al., 2015a) or mimosine (Mori et al., 2015) at $100 \mu \mathrm{M}$. Not only the endogenous contents of allelochemicals in the protoplasts themselves, but also the uptake efficiency of these chemicals from culture media and the cellular mechanisms of inhibition on lettuce protoplasts growth might be considered.

This is also the first report of an anthocyanin with strong allelopathic activity identified as cyanin. The antioxidant and antimicrobial activities of anthocyanins have been studied (Kähkönen \& Heinonen, 2003; Cisowska et al., 2011; Takeda et al., 2013), but not the allelopathic activities of anthocyanins. We have been studying the allelopathic activities of chemicals containing the cyanidin-structure using the protoplast co-culture method (Sasamoto et al., 2017d).

We found the protoplast co-culture method with digital image analysis useful to study the allelopathic activities of chemicals in lettuce protoplasts at three different growth stages. Though the yellow color accumulation is observed only during the growth of lettuce protoplasts, the protoplast co-culture method can be applied not only using lettuce as a recipient plant, but also neighboring plants in the field (Sasamoto et al., 2013).

\section{Acknowledgments}

H.S. thanks Mr. Y. Sasamoto for his help in digital image analysis.

\section{References}

Azumi, Y., \& Sasamoto, H. (2017). Production of protoplasts from pollen mother cells of higher plants and observation of their chromosomes. Science Journal of Kanagawa University, 28, 85-92.

Cisowska, A., Wojnicz, D., \& Hendrich, A. B. (2011). Anthocyanins as antimicrobial agents of natural plant origin. Natural Product Communications, 6, 149-156.

Fujii, Y. (2000). Allelopathy, Nobunkyo, Tokyo.

Fujise, K., Yokota, S., \& Sasamoto, H. (2018). Evaluation of allelochemicals, abscisic acid and coumarin, in leaf-origin suspension cultured cells of Prunus yedoensis using protoplast co-culture bioassay method. American Journal of Plant Sciences, 9, 172-184. https://doi.org/10.4236/ajps.2018.92015

Hasegawa, A. (2014). Research on the relationship between salts tolerance and allelopathy at the cellular level in mangrove plants. Ph.D. thesis of Yokohama National University. pp 1-124.

Hasegawa, A., Kurita, A., Hayashi, S., Fukumoto, T., \& Sasamoto, H. (2013). Halophilic and salts tolerant protoplast cultures of mangrove plants, Sonneratia alba and Avicennia alba, Plant Biotechnology Reports, 7, 205-209. https://doi.org/10.1007.s11816-012-0251-2

Hasegawa, A., Oyanagi, T., Minagawa, R., Fujii, Y., \& Sasamoto, H. (2014) An inverse relationship between allelopathic activity and salt tolerance in suspension cultures of three mangrove species, Sonneratia alba, $S$. caseolaris and $S$. ovata: Development of a bioassay method for allelopathy, the protoplast co-culture method. Journal of Plant Research, 127, 755-761. https://doi.org/10.1007/s10265-014-0651-1

Hayashi, K. (1933). Vereinfachte Darstellungsmethode der Anthocyaninpräparate aus Dahlienblüten. The Botanical Magazine, Tokyo, 47, 394-399.

Hayashi, S., Kuriyama, S., Kawana, Y., Hasegawa, A., Kurita, A., Minagawa, R., \& Sasamoto, H. (2009). Stimulatory effects of sea salts on cell growth in liquid culture of Avicenniaceae mangrove. Plant Biotechnology, 26, 561-564. https://doi.org.10.5511.plantbiotechnology.26.561

Inoue, A., Mori, D., Minagawa, R., Fujii, Y., \& Sasamoto, H. (2015). Allelopathy in a leguminous mangrove plant, Derris indica: Protoplast co-culture bioassay and rotenone effect. Natural Product Communications, 5, 747-750.

Kähkönen, M. P., \& Heinonen, M. (2003). Antioxidant activity of anthocyanins and their aglycons. Journal of Agricultural and Food Chemistry, 51, 628-633. https://doi.org/10.1021/jf025551i

Kawana, Y., Yamamoto, R., Mochida, Y., Suzuki, K., Baba, S., \& Sasamoto, H. (2007). Generation and maintenance of suspension cultures from cotyledons and their organogenic potential of two mangrove species, Sonneratia alba and S. caseolaris. Plant Biotechnology Report, 1, 219-226. 
https://doi.org/10.1007.s11816-007-0035-2

Kawana, Y., \& Sasamoto, H. (2008). Stimulation effects of salts on growth in suspension culture of a mangrove plant, Sonneratia alba, compared with another mangrove, Bruguiera sexangula and non-mangrove tobacco BY-2 cells. Plant Biotechnology, 25,151-155. https://doi.org.10.5511.plantbiotechnology.25.151

Minagawa, R., Zhang, W., Sun, Y., Zhou, S., \& Kondo, K. (2016). Molecular phylogeny of Sonneratia. Chromosome Botany, 11(3), 56-60. https://doi.org/10.3199/iscb.11.56

Mori, D., Ogita, S., Fujise, K., Inoue, A., \& Sasamoto, H. (2015). Protoplast co-culture bioassay for allelopathy in leguminous plants, Leucaena leucocephala and Mucuna gigantea, containing allelochemical amino acids, mimosine and L-DOPA. Journal of Plant Studies, 4, 1-11. https://doi.org/10.5539/jps.v4n1p1

Murashige, T., \& Skoog, F. (1962). A revised medium for rapid growth and bio assays with tobacco tissue cultures. Physiologia Plantarum, 15, 473-497. https://doi.org/10.1111/j.1399-3054.1962.tb08052.x

Ogawa, K., Sakakibara, H., Iwata, R., Ishii, T., Sato, T., Goda, T., Shimoi, K., \& Kumazawa, S. (2008). Anthocyanin composition and antioxidant activity of the crowberry (Empetrum nigrum) and other berries. Journal of Agricultural and Food Chemistry, 56(12), 4457-4462. https://doi.org/10.1021/jf800406v

Ogita, S., \& Sasamoto, H. (2017). In vitro bioassay of allelopathy in four bamboo species; Bambusa multiplex, Phyllostachys bambusoides, P. nigra, Sasa kurilensis, using sandwich method and protoplast co-culture method with digital image analysis. American Journal of Plant Sciences, 8, 1699-1710. https://doi.org/10.4236/ajps.2017.87117

Rasband, W. S. (1997-2016). Image J.U.S. National Institute of Health, Bethesda, Maryland, USA. http://imagej.nih.gov/ij

Sasamoto, H., \& Ashihara, H. (2014). Effect of nicotinic acid, nicotinamide and trigonelline on the proliferation of lettuce cells derived from protoplasts. Phytochemistry Letters, 7, 38-41. https://doi.org/10.1016/j.phytol.2013.09.008

Sasamoto, H., Wakita, Y., \& Baba, S. (1997). Effect of high sorbitol concentration on protoplast isolation from cotyledons of mangroves, Avicennia marina, and A. lanata. Plant Biotechnology, 14, 101-104. http://dx.doi.org/10.5511/plantbiotechnology.14.101

Sasamoto, H., Murashige-Baba, T., Inoue, A., Sato, T., Hayashi, S., \& Hasegawa, A. (2013). Development of a new method for bioassay of allelopathy using protoplasts of a leguminous plant Mucuna pruriens with a high content of the allelochemical L-DOPA. Journal of Plant Studies, 2, 71-80. https://doi.org/10.5539/jps.v2n2p71

Sasamoto, H., Fujii, Y., \& Ashihara, H. (2015a). Effect of purine alkaloids on the proliferation of lettuce cells derived from protoplasts. Natural Product Communications, 5, 751-754.

Sasamoto, H., Murashige-Baba, T., Sato, T., Hasegawa, A., Wasano, N., \& Fujii, Y. (2015b). Development of a simple high-throughput bioassay method for allelopathy: Protoplast co-culture with image analysis. Abstracts of the 2015 in Vitro Biology Meeting. In Vitro Cellular \& Developmental Biology-Plant, 51, 12.

Sasamoto, H., Azumi, Y., \& Suzuki, S. (2017a). Development of a high-throughput bioassay method of allelopathy-protoplast co-culture and digital image analysis. Science Journal of Kanagawa University, 28, 63-70.

Sasamoto, H., Azumi, Y., Shimizu, M., Hachinohe, Y., \& Suzuki, S. (2017b). In vitro bioassay of allelopathy of Arabidopsis thaliana by sandwich method and protoplast co-culture method with digital image analysis. Plant Biotechnology, 34, 199-202. https://doi.org/10.5511/plantbiotechnology.17.1204a

Sasamoto, H., Iwashina, T., Suzuki, S., Azumi, Y., \& Fujii, Y. (2017c). Evaluation of allelopathic activities of red callus of Sonneratia ovata containing an anthocyanin, cyanidin-3,5-di- $O$-glucoside, using protoplast co-culture method. Abstracts of the 23th Annual Meeting of the Japan Society for Mangroves, $\mathrm{p} 5$.

Sasamoto, H., Suzuki, S., Nakagawa, W., Sasamoto, Y., Wasano, N., \& Fujii, Y. (2017d). Allelopathic activities of anthocyanin-accumulating transformant of Spiraea cantoniensis. Proceedings of the 81th Annual Meeting of the Botanical Society of Japan, p 250.

Spalding, M., Kainuma, M., \& Cocking, E. C. (2010). World Atlas of Mangroves. Earthscan, UK and USA.

Takeda, K., Saito, N., \& Iwashina, T. (Eds.) (2013). Plant Pigments, Flavonoids. Bun-ichi Co. Ltd., Tokyo. ISBN978-4-8299-6521-4. 
Takemura, T., Sakuno, E., Kamo, T., Hiradate, S., \& Fujii, Y. (2013). Screening of the growth-inhibitory effects of 168 plant species against lettuce seedlings. American Journal of Plant Sciences, 4, 1095-1104. https://doi.org/10.4236/ajps.2013.45136

Tomlinson, P. B. (1986). The Botany of Mangroves. Cambridge University Press, New York.

Tsuchiya, S., Ogita, S., Kawana, Y., Oyanagi, T., Hasegawa, A., \& Sasamoto, H. (2013). Relation between amino acids profiles and recalcitrancy of cell growth or salt tolerance in tissue and protoplast cultures of three mangrove species, Avicennia alba, Bruguiera sexangula, and Sonneratia alba. American Journal of Plant Sciences, 4, 1366-1374, https://doi.org/10.4236/ajps.2013.47167

Wu, S.-B., Wen, Y., Li, X.-W., Zhao, Y., Zhao, Z., \& Hu, J.-F. (2009). Chemical constituents from the fruits of Sonneratia caseolaris and Sonneratia ovata (Sonneratiaceae). Biochemical Systematics and Ecology, 37, 1-5. https://doi.org/10.1016/j.bse.2009.01.002

Yamamoto, R., Kawana, Y., Minagawa, R., \& Sasamoto, H. (2011). Effects of sea salts on induction of cell proliferation in liquid cultures of mangrove plants, Sonneratia caseolaris and S. alba. American Journal of Plant Sciences, 2, 35-42, https://doi.org/10.4236/ajps.2011.21004

\section{Copyrights}

Copyright for this article is retained by the author(s), with first publication rights granted to the journal.

This is an open-access article distributed under the terms and conditions of the Creative Commons Attribution license (http://creativecommons.org/licenses/by/4.0/). 